\title{
Optimized power allocation for iterative multiuser detection for a SC-CDMA uplink
}

\author{
P. Weitkemper ${ }^{1}$, K. Zielinski ${ }^{2}$, K.-D. Kammeyer ${ }^{1}$, and R. Laur ${ }^{2}$ \\ ${ }^{1}$ Department of Communications Engineering, University of Bremen, Otto-Hahn-Allee, 28359 Bremen, Germany \\ ${ }^{2}$ Institute for Electromagnetic Theory and Microelectronics, University of Bremen, Otto-Hahn-Allee, 28359 Bremen, \\ Germany
}

\begin{abstract}
This paper deals with optimization of the received power profile for iterative parallel and successive interference cancellation (PIC/SIC) in coded CDMA systems. For practical implementation additional constraints should be applied. This paper focuses on the maximum tolerable bit error rate. It will be shown that optimized power profile can considerably gain the overall system performance. Due to unequal required receive powers an allocation to certain users can be done with respect to their individual power constraints. This is important especially in near-far scenarios. Beside these constraints also the maximum number of iterations is implemented due to limiting the computational complexity in the receiver.
\end{abstract}

\section{Introduction}

Nonlinear iterative multi-user detection exploiting channel decoding is a well known approach to increase the possible system load while still achieving single-user performance. Analysis of the PIC scheme has been done by different approaches (Schlegel and Shi, 2004; Boutros and Caire, 2002; Kuehn, 2004) and the possibility of power profile optimization has been shown in e.g. Caire et al. (2004). So far optimization of receive power distribution was done with a simple optimization goal and only few constraints. The focus was on minimizing the sum over all received powers which may be feasable for downlink but not for uplink scenarios. The constraint was ensuring all users to have a maximum tolerable bit error rate which is the most interesting point in practicle systems, but single user performance is not required implicitly. If the SINR is still higher than the SNR but sufficiently small for the desired BER, there is no need for increasing the power further only to reach this certain

Correspondence to: P. Weitkemper

(weitkemper@ant.uni-bremen.de) point. In the uplink of a cellular network, individual power constraints have to be considered. One possibility is to assume additional constraints for individual powers of the users which was presented in Weitkemper et al. (2006). A more general approach used in this paper is not to minimize the sum over all powers but the maximum power which results in a more homogeneous distribution. Furthermore some additional constraints are considered which take practical implemetation issues into account e.g. maximum number of iterations. For successive interference cancellation the analysis is more difficult because the statistics of the users differ from each other. The generalization of the analysis to SIC and the basics of power profile optimization were presented in Weitkemper et al. (2005).

The paper is organized as follows: Sect. 2 introduces the system model of the considered CDMA system. In Sect. 3 the analysis based on multi-user efficiency (MUE) (Boutros and Caire, 2002) is described and applied to the parallel interference cancellation. The generalized MUE analysis of SIC is given in Sect. 4. In Sect. 5 power profile optimization is introduced for both detection schemes. Results for different optimization objects and additional constraints are presented and discussed in Sect. 6. A conclusion is given in Sect. 7.

\section{System model}

In order to simplify derivations and notation we assume a synchronous single carrier- (SC-) CDMA system with a complex AWGN channel and pseudo-noise spreading sequences (Verdú, 1998). The number of active users is denoted by $U$. The information bit vector of the $u$-th user is denoted by $\mathbf{d}_{u}$, which is encoded by a convolutional code of rate $R_{c}=1 / 2$ that is identical for all users. The coded bit sequence is BPSK-modulated and interleaved by a user-specific random interleaver $\Pi_{u}$ of length $L$. Finally the signals are spread with random spreading codes $s_{u}(k) \in\{-1 / \sqrt{N},+1 / \sqrt{N}\} . k$ 


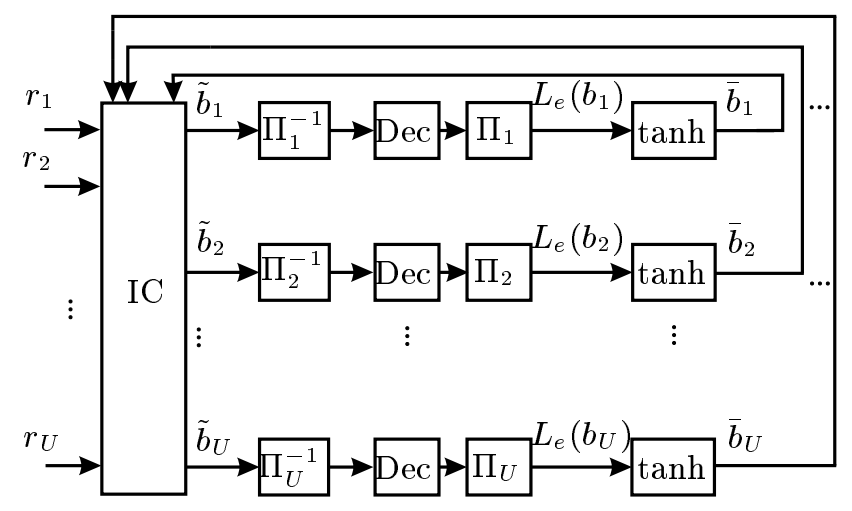

Fig. 1. Receiver structure of PIC.

denotes the chip and $l$ the symbol index. The length $N$ of the sequences $s_{u}(k)$ is called spreading factor and $\beta=U / N$ denotes the system load. $\mathbf{b}$ is assumed to be the vector comprising BPSK symbols of all users at a particular time instance and $\mathbf{C}$ is the $N \times U$ spreading matrix. It contains the vectors of spreading sequences as columns each multiplied with an individual phase term of the channel. The received vector containing the superposition of the spread signals of all users and the noise can be described in vector-matrix notation, yielding

$$
\mathbf{y}=\mathbf{C b}+\mathbf{n} \text {. }
$$

For notational simplicity time indices have been dropped. The vector $\mathbf{n}$ represents the complex additive white Gaussian noise with covariance matrix $\sigma_{n}^{2} \mathbf{I}$. At the receiver a bank of matched filters (MF) is applied for despreading and the realvalued matched filter output can be written as

$$
\mathbf{r}=\operatorname{Re}\left\{\mathbf{C}^{H} \mathbf{y}\right\}=\underbrace{\operatorname{Re}\left\{\mathbf{C}^{H} \mathbf{C}\right\}}_{\mathbf{R}} \mathbf{b}+\underbrace{\operatorname{Re}\left\{\mathbf{C}^{H} \mathbf{n}\right\}}_{\tilde{\mathbf{n}}} .
$$

The elements of $\mathbf{R}$ contain the real part of the correlation coefficients $\varrho_{i j}=\operatorname{Re}\left\{\rho_{i j}\right\}$ between the $i$-th and the $j$-th user's signature sequence with $\mathrm{E}\left\{\left|\rho_{i j}\right|\right\}_{i \neq j}=1 / N$. The multi-user interference characterized by these correlation coefficients degrades the performance significantly even for moderate system loads if individual decoding and hard decision is applied to these matched filter outputs. Interference cancellation techniques are able to improve performance significantly by estimating the multi-user interference and cancelling it before detection. Additional gain is achieved by iterative structures.

\section{Multi-user efficiency}

Figure 1 shows the structure of a parallel interference canceller. In order to get at least approximated extrinsic loglikelihood-ratios $L_{e}\left(b_{u}\right)$ at low computational cost, the MaxLog-MAP channel decoder is applied. The soft estimates

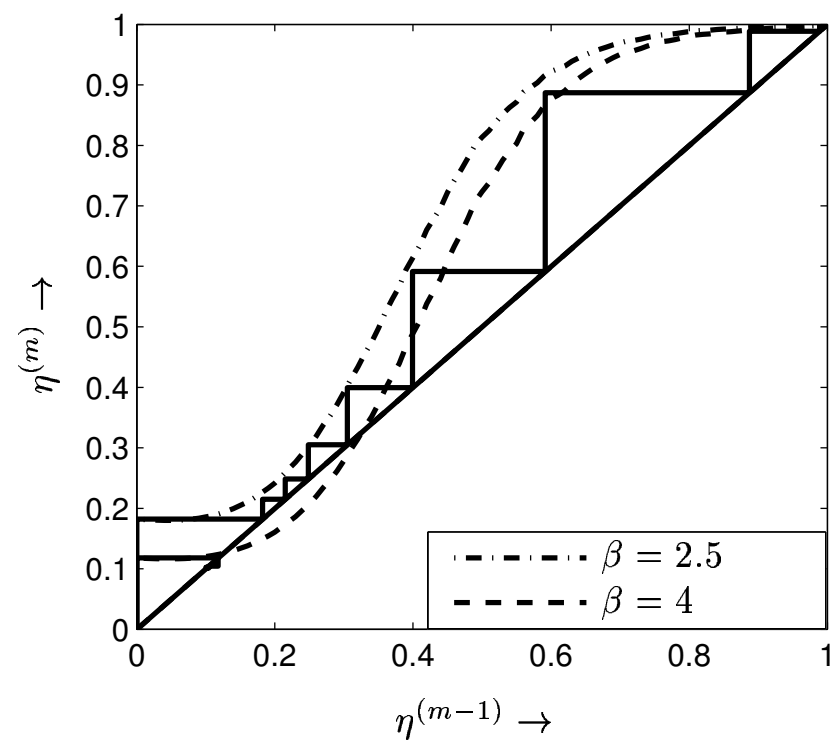

Fig. 2. Predicted transfer functions and trajectories for PIC, $N=4$, $U=10 / 16, E_{b} / N_{0}=6 \mathrm{~dB}$.

of the coded symbols $\bar{b}$ are calculated as $\bar{b}=\tanh \left(L_{e} / 2\right)$. The signal-to-interference-plus-noise-ratio (SINR) of each branch is a parameter indicating the quality of the interference cancellation and is defined as $\operatorname{SINR}=2 \sigma_{d}^{2} /\left(\sigma_{n}^{2}+\sigma_{\text {MUI }}^{2}\right)$. It is equal to the $\mathrm{SNR}=2 E_{S} / N_{0}$ in the case of perfect interference cancellation which is equivalent to the single-user bound (SUB). $\sigma_{d}^{2}$ and $\sigma_{\mathrm{MUI}}^{2}$ describe the variance of the desired signal and of the remaining multi-user interference after cancellation respectively. The latter can be calculated as $\sigma_{\text {MUI }}^{2}=\sigma_{d}^{2} \cdot \mu(U-1) / N$. The remaining mean squared error of the estimated symbols after decoding is denoted as $\mu=\mathrm{E}\left\{|\bar{b}-b|^{2}\right\}$ which is approximately the same for each user in the case of PIC. The ratio of SINR and SNR is called multi-user efficiency (MUE) and is denoted by $\eta$ (Boutros and Caire, 2002). Perfect interference cancellation is indicated by $\eta=1$ and therefore describes the case with no loss compared to the SUB. For the large system limit $(N, U \rightarrow \infty)$ $(U-1) / N \approx U / N=\beta, \eta$ can be written as

$$
\eta=\frac{\mathrm{SINR}}{\mathrm{SNR}}=\frac{2 \sigma_{d}^{2} /\left(\sigma_{n}^{2}+\sigma_{\mathrm{MUI}}^{2}\right)}{2 \sigma_{d}^{2} / \sigma_{n}^{2}}=\frac{1}{1+\beta \mu E_{s} / N_{0}}
$$

The parameter $\eta$ can be used to visualize and predict the behavior of an iterative detection scheme. In the initial iteration there is no a-priori information available for interference cancellation. The soft bits are initialized with zero, the variance $\mu$ is therefore equal to 1 and the MUE becomes $\eta^{(1)}=1 /\left(1+\beta E_{s} / N_{0}\right)$. After simultaneously decoding all users, soft estimates $\bar{b}$ of the transmitted symbols are obtained which are used in the next iteration for interference cancellation. Since channel decoding is generally a nonlinear process $\mu$ cannot be calculated analytically, but has to be predetermined. The output error $\mu^{(m)}$ of the decoder in the 


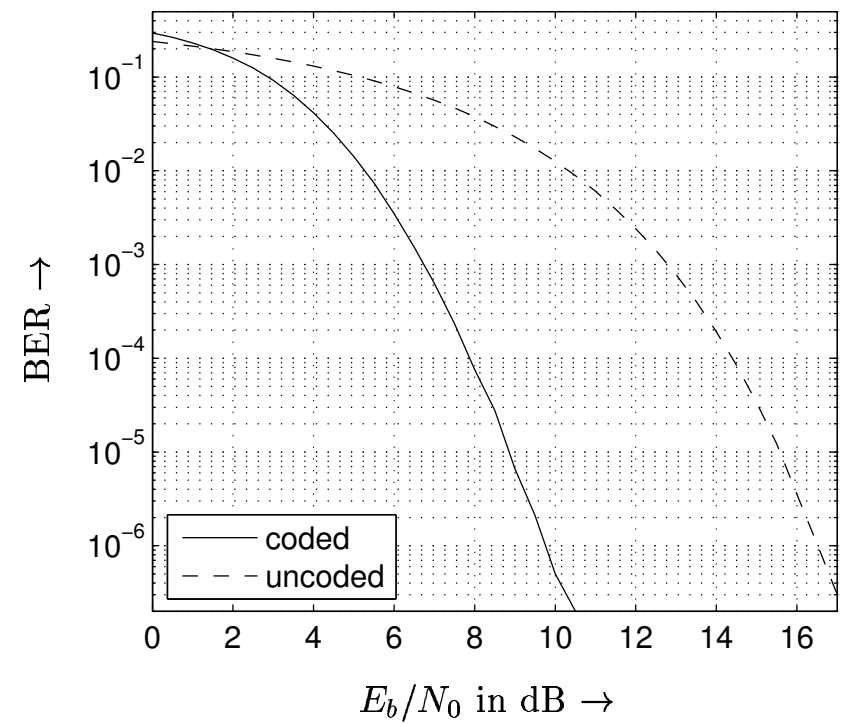

Fig. 3. Coded and uncoded error rate for a AWGN channel and [7 5] convolutional code.

$m$-th iteration depends on the SINR at the input

$$
\mu^{(m)}=g(\mathrm{SINR})=g\left(\eta^{(m-1)} \mathrm{SNR}\right)
$$

and therefore on the MUE of the previous iteration $\eta^{(m-1)}$. Because $\eta^{(m)}$ itself depends on $\mu^{(m)}$ the behavior of the PIC at iteration $m$ can be described by $\eta^{(m)}=f\left(\eta^{(m-1)}\right)$. This function is depicted in a two-dimensional plot in Fig. 2. The transfer function describes the theoretical behavior and the trajectory gives the measured values during simulation. The detection starts in the lower left corner and tends to the upper right corner. This point corresponding to $\eta=1$ describes perfect interference cancellation. It can be seen that the behavior is predicted very precisely. This plot corresponds to a system with a spreading factor $N=4, U=10 / 16$ users, an $E_{b} / N_{0}$ of $6 \mathrm{~dB}$ and a convolutional code with generator polynomials $[7,5]_{8}$. The system with 10 users will converge to the SUB within 7 iterations. In the case of 16 users there is an intersection between the transfer function and the bisecting line so the detection gets stuck at $\eta \approx 0.15$.

Since multi-user efficiency is a relative quantity, the performance assuming the uncoded BER has to be calculated by

$$
P_{b}=\frac{1}{2} \cdot \operatorname{erfc}\left(\sqrt{\frac{\mathrm{SINR}}{2}}\right)=\frac{1}{2} \cdot \operatorname{erfc}\left(\sqrt{\frac{P}{2 \sigma_{n}^{2}}}\right)
$$

$\eta$ only includes information about the error probability before channel decoding. As the coded bit error rate is the important parameter for QoS requirements, a connection between these error probabilities have to be considered. The coded and uncoded BER for the [7 5] code is shown in Fig. 3.
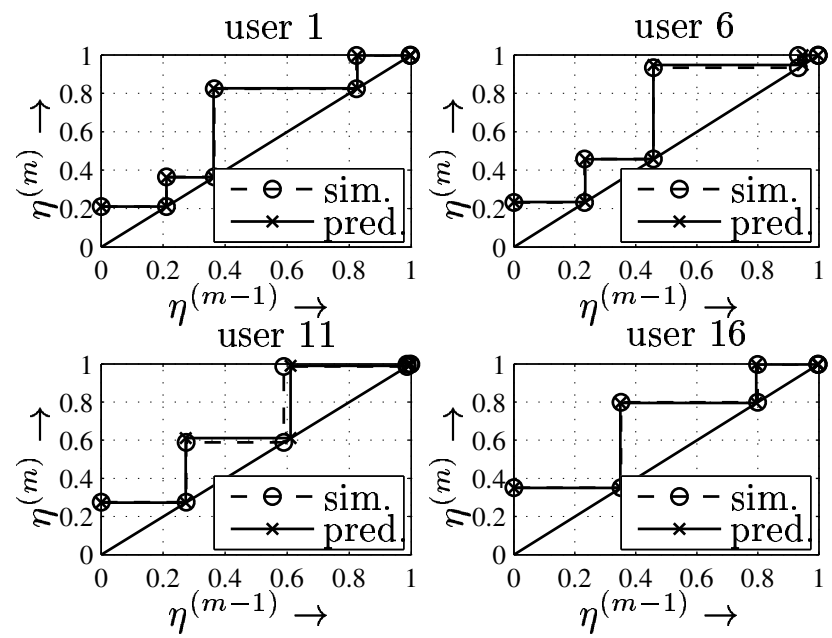

Fig. 4. Predicted and simulated trajectories for SIC, $N=8, U=16$ and $E_{b} / N_{0}=6 \mathrm{~dB}$.

\section{Analysis of successive interference cancellation}

The difference concerning successive interference cancellation is the processing of the users one after another. The parameters change not only after 1 iteration but during the iterations when decoding each individual user. Therefore the prediction in the same manner as for PIC is not possible. While for PIC the error variance $\mu$ is the same for all users in the large system limit, this is not the case for SIC. The $U$ users have different variances $\mu_{u}^{(m)}$ at each iteration $m$. The remaining errors of the users are assumed to be independent. So a simple addition of their variances weighted with the corresponding correlation coefficient can be applied for calculating the resulting interference on the desired user signal. For that reason the MUE can be calculated by

$$
\eta_{u}^{(m)}=\frac{1}{1+\frac{1}{N}\left(\sum_{i=1}^{u-1} \mu_{i}^{(m)}+\sum_{i=u+1}^{U} \mu_{i}^{(m-1)}\right) \frac{E_{s}}{N_{0}}} .
$$

To show how good the prediction works, Fig. 4 depicts the predicted and the simulated trajectories for the same system parameters as in Fig. 2 in one diagram per user. It can be seen that the prediction works well also in the successive interference cancellation case. A simple transfer function as shown in Fig. 2 cannot be drawn in these plots since the transfer function differs for each user and each iteration due to being conditioned on the current state of all the other users. In order to avoid a very complex diagram only the trajectories are depicted in Fig. 4. 


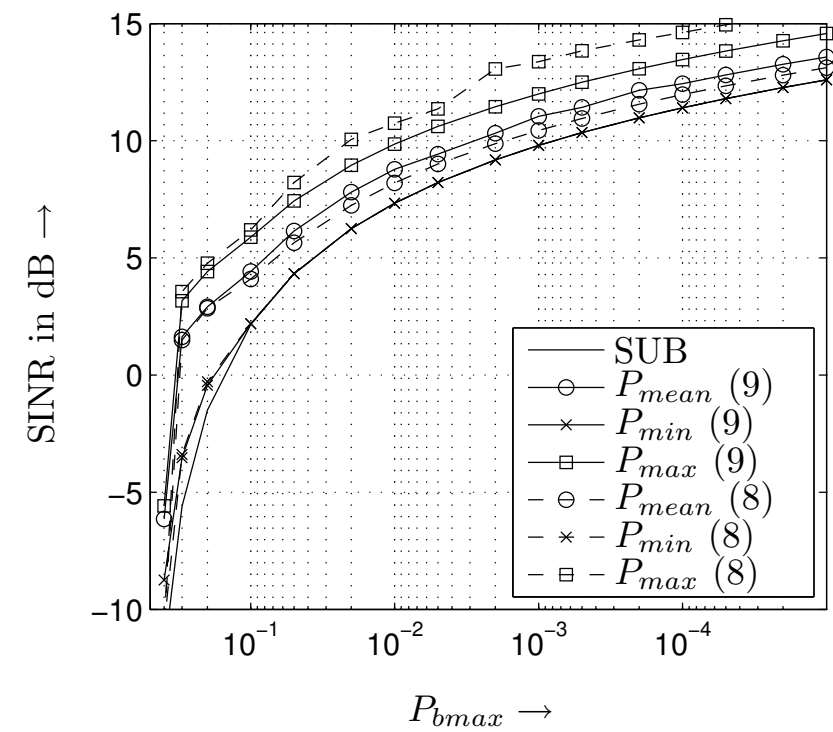

Fig. 5. Required receive power of all users dependent on the required BER for different expressions to be minimized (Eqs. 8 and 9).

\section{Power profile optimization}

\subsection{Formulation of the optimization problem}

Up to now the analysis was based on uniformly distributed powers of the users. To describe an unequal power distribution by multi-user efficiency, a way to calculate a kind of average efficiency is necessary (Schlegel and Shi, 2004). Thus the resulting multi-user efficiency can be calculated more generally as

$$
\eta_{u}=\frac{1}{1+\frac{\bar{E}_{s}}{N_{0}} \frac{1}{N} \sum_{v \neq u} \mu_{v} \cdot P_{v}} \quad, \quad \sum_{v} P_{v}=U .
$$

$\bar{E}_{S} / N_{0}$ is defined as an average value over all users in order to get an appropriate criterion for fair comparison with the equal power case. The criterion for convergence is still reaching the point of $\eta=1$ after a finite number of iterations. For the PIC this is fulfilled if $f(\eta) \geqslant \eta, \eta \in[0,1]$. The number of iterations needed depends on the width of the tunnel. Whether the tunnel is open or not depends also on the power distribution. For PIC it turns out that equal power for all users is not the best choice, as presented e.g. in Schlegel and Shi (2004) and Caire et al. (2004). The optimization problem for PIC can be described by Caire et al. (2004)

$$
\min _{P_{1}, . ., P_{U}} \sum_{u} P_{u} \quad \text { s. t. }\left\{\begin{array}{l}
f(\eta) \geqslant \eta+\varepsilon, \eta \in[0 ; 1] \\
P_{u}>0 \quad, \quad u=1, \ldots U
\end{array}\right.
$$

if the sum of all received powers should be minimized and single user performance for all users is claimed. These con-

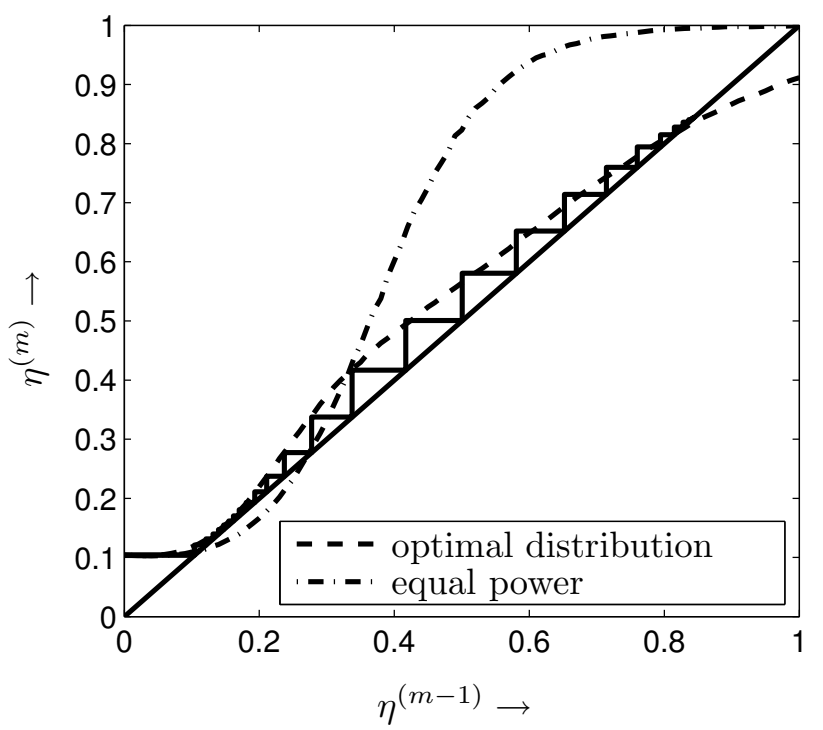

Fig. 6. Transfer function with optimized power profile for $P_{b \text { max }}=0.2$ and $\beta=4$.

ditions can be adapted to the particular need. For example in this work it will be

$$
\min _{P_{1}, \ldots, P_{U}} \max _{u} P_{u} \text { s.t. }\left\{\begin{array}{l}
P_{b}(u) \leqslant P_{b} \max \forall u=1, \ldots U \\
N_{i t} \leqslant N_{i t} \max \forall u=1, \ldots U \\
P_{u}>0 \forall u=1, \ldots U
\end{array}\right.
$$

For taking a maximum number of iterations into account, not the transfer function but the trajectory is used for the first constraint. After each iteration up to the maximum the MUE for all users is calculated and either this value or the corresponding BER is considered. For the SIC a similar expression is used although the calculation of $\eta$ is different (Eq. 6).

\subsection{Differential evolution}

The cost functions of the examined optimization problems contain many local optima (multimodal functions), especially for SIC. Furthermore, the search space is highly constrained. Therefore a starting point in the vicinity of the global optimum would be required for the employment of local optimization techniques. As the global optimum is not known in advance global optimization methods have to be used. In this paper the Differential Evolution (DE) algorithm is chosen that belongs to the class of evolutionary algorithms (Price, 1999). As the name indicates evolutionary algorithms are motivated by the natural evolution process. In contrast to local search algorithms not only one but several points (population) in the search space are regarded at a given time instance. The advantage is that evolutionary algorithms do not need any knowledge of the search space. Differential Evolution furthermore possesses the property of fast convergence 


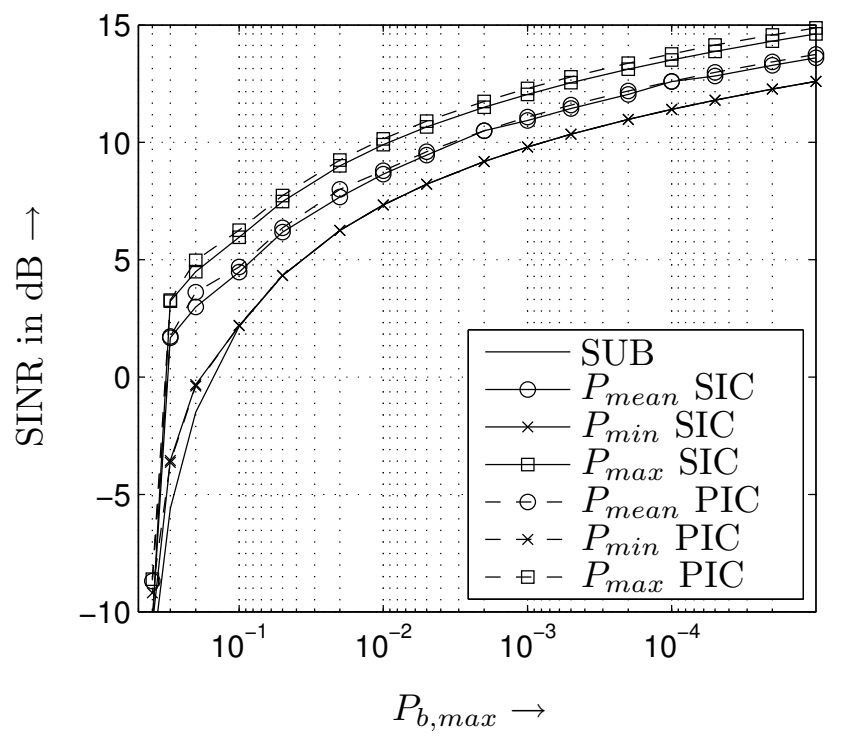

Fig. 7. Required receive power of all users dependent on the required BER for SIC and PIC, $\beta=4,20$ iterations, mimimized according to Eq. (9).

due to an adaptive step size. Additionally, it is easy to use because of the few control parameters.

The starting population is generated randomly and should be sufficiently large for a high diversity. In this case a population size of $N P=80$ is used. Standard settings are used for other control parameters (Zielinski et al., 2005a). Population members of the current population are combined using the evolutionary operators mutation, recombination and selection to generate the next generation until a stopping condition is fulfilled. In this case the distribution of population members is monitored and the algorithm terminates when the maximum distance of every individual to the best population member is beneath a threshold (Zielinski et al., 2005b). In Section 6 the number of generations is restricted to 1000 due to limited computational resources.

\section{Optimization results}

As noted before in this paper not the sum of all powers is minimized but the maximum power. This seems to be more sufficient for cellular networks. Consequently, the overall power maybe increased but in the uplink the individual power constraint is the limiting point. In Fig. 5 are minimum, maximum and mean powers of the optimized profile depicted, which were optimized for different maximum uncoded BER. The lower bound for the receive power is the single user case. In this figure the difference between the optimization criteria (8) and (9) can be observed. The mean power is lower in the case of Eq. (8) which was the aim of this approach. But the maximum required power is up to $1 \mathrm{~dB}$ higher than for approach (9) over the whole BER range.

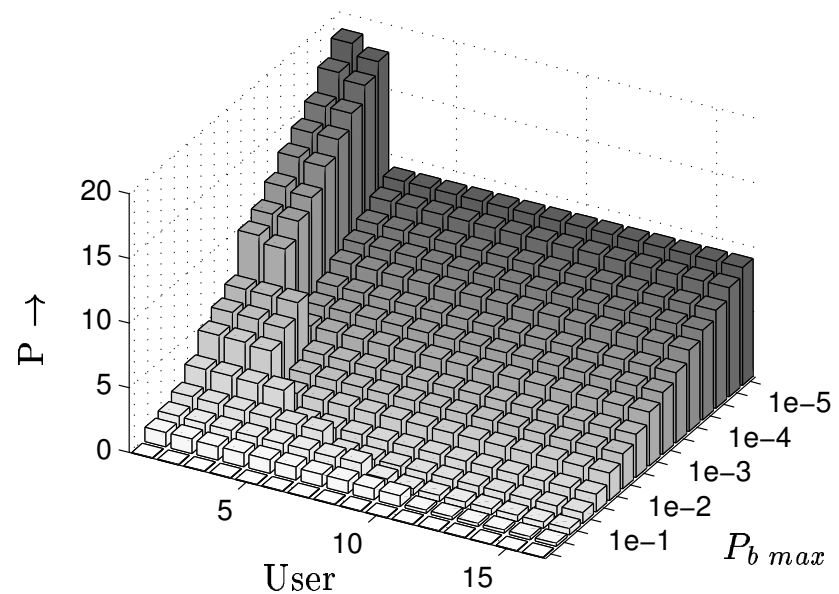

Fig. 8. Power profiles for different $P_{b}$ max assuming Eq. (8).

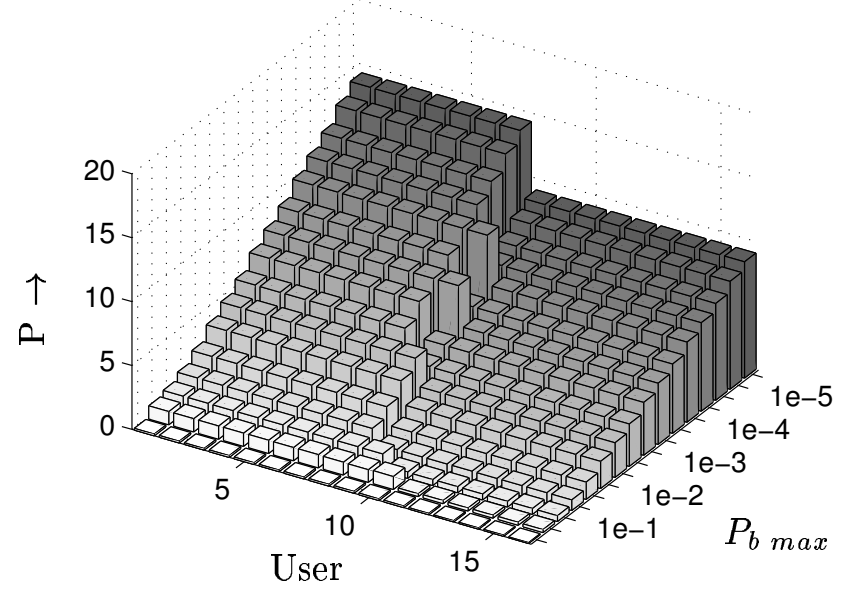

Fig. 9. Power profiles for different $P_{b}$ max assuming Eq. (9).

Results for the equal power case are not depicted, because the considered bit error rates will not be reached in this case. Just up to a load of $\approx 3$ equal power systems may reach SUB.

The minimum power is in most cases equal to the minimum power for the single user case. Only for very low BERs this is no longer the case. This means on the other hand, for a given receive power the single user performance is not reached. This is due to the more general constraint only assuming the BER not the convergence to SUB. In Fig. 6 this case is illustrated with the corresponding transfer function. The iterative scheme gets stuck at $\eta \approx 0.8$. This plot corresponds to a uncoded target errror rate of 0.2. The results for PIC and SIC are compared in Fig. 7. As already noted in Weitkemper et al. (2005) SIC converges faster than PIC. With the additional constraint of a limited number of iterations which is also applied in this paper, this fact results in a decreased power. This effect gets smaller the more iterations are assumed. The resulting power profiles for different values of $P_{b}$ max are depicted in Fig. 8 for the case of 
minimizing the sum of all powers. It can be seen that with decreasing error rate the number of users having a higher power than for the single user case gets smaller. But the ammount of power required for these users grows disproportionate to the minimum power. This behavior fulfills the requirement of minimum overall receive power, but in practice these high powers may not be possible. In Fig. 9 the power distributions with minimizing the maximum power can be seen. The number of users having an increased power is much higher than in Fig. 8 but the absolute maximum is smaller. In general the distribution gets more homogeneous. This distribution is more appropriate to practical cellular networks. In consideration of near-far effects the powers can be allocated to the users depending on their individual power constraint. For PIC the allocation order has no influence on the system performance and for SIC the detection order can be adapted if necessary. But as already observed in Weitkemper et al. (2005) the order of detection has minor influence at these high loads.

Any combination of individual and total power constraint can be implemented very easy. Even specific maximum supportable power by certain users can be taken into account.

\section{Conclusions}

In this paper some aspects of power profile optimization were investigated. The maximum bit error rate was considered as main constraint. At low BER this constraint gives different results than claiming convergence to the SUB. Additionally the expression to be minimized was adapted to the scenario of uplink communiaction in cellular networks. Not the overall power but the individual power is strictly limited and should be saved. So the optimization focusses on minimizing the maximum power. The results differ as well in general shape of the profiles as in the required maximum and mean power. Furthermore limited number of iterations was taken into account due to the limited time and resources for decoding in the base station. All these aspects deliver an optimization suitable for practicle system requirements.

\section{References}

Boutros, J. and Caire, G.: Iterative Multiuser Joint Decoding: Unified Framework and Asymptotic Analysis, IEEE Transactions on Information Theory, 48, 1772-1793, 2002.

Caire, G., Mueller, R., and Tanaka, T.: Iterative Multiuser Joint Decoding: Optimal Power Allocation and Low-Complexity Implementation, IEEE Transactions on Information Theory, 50, 19501973, 2004.

Kuehn, V.: Analysis of Iterative Multi-User Detection Schemes with EXIT Charts, in: IEEE International Symposium on Spread Spectrum Techniques and Applications (ISSSTA), Sydney, Australia, 2004.

Price, K.: An Introduction to Differential Evolution, in: New Ideas in Optimization, edited by: Corne, D., Dorigo, M., and Glover, F., pp. 79-108, McGraw-Hill, London, 1999.

Schlegel, C. and Shi, Z.: Optimal Power Allocation and Code Selection in Iterative Detection of Random CDMA, in: Zurich Seminar on Digital Communications, 2004.

Verdú, S.: Multiuser Detection, Cambridge University Press, 1998.

Weitkemper, P., Kühn, V., and Kammeyer, K.-D.: Analysis of Iterative Successive Interference Cancellation in SC-CDMA Systems, in: Fifth International Workshop on Multi-Carrier SpreadSpectrum (MC-SS 2005), Oberpfaffenhofen, Germany, 2005.

Weitkemper, P., Zielinski, K., Kammeyer, K.-D., and Laur, R.: Optimization of Interference Cancellation in Coded CDMA Systems by Means of Differential Evolution, in: 4th International Symposium on Turbo Codes \& Related Topics, Munich, Germany, 2006.

Zielinski, K., Peters, D., and Laur, R.: Constrained Multi-Objective Optimization Using Differential Evolution, in: Third International Conference on Computational Intelligence, Robotics and Autonomous Systems, Singapore, 2005a.

Zielinski, K., Peters, D., and Laur, R.: Stopping Criteria for SingleObjective Optimization, in: Third International Conference on Computational Intelligence, Robotics and Autonomous Systems, Singapore, 2005b. 